\title{
Type Ibc supernovae in disturbed galaxies: evidence for a top-heavy IMF
}

\author{
S M Habergham ${ }^{1}$, J P Anderson ${ }^{2}$ and P A James ${ }^{1}$ \\ ${ }^{1}$ Astrophysics Research Institute, Liverpool John Moores University, Twelve Quays \\ House, Birkenhead, CH41 1LD \\ ${ }^{2}$ Departamento de Astronomía, Universidad de Chile, Casilla 36-D, Santiago, Chile \\ E-mail: smh@astro.livjm.ac.uk
}

\begin{abstract}
We compare the radial locations of 178 core-collapse supernovae to the $R$-band and $\mathrm{H} \alpha$ light distributions of their host galaxies. When the galaxies are split into 'disturbed' and 'undisturbed' categories, a striking difference emerges. The disturbed galaxies have a central excess of core-collapse supernovae, and this excess is almost completely dominated by supernovae of types $\mathrm{Ib}$, Ic and $\mathrm{Ib} / \mathrm{c}$, whereas type II supernovae dominate in all other environments. The difference cannot easily be explained by metallicity or extinction effects, and thus we propose that this is direct evidence for a stellar initial mass function that is strongly weighted towards high mass stars, specifically in the central regions of disturbed galaxies.
\end{abstract}

Keywords: galaxies:interactions-galaxies:ISM-supernovae:general 


\section{Introduction}

Following the pioneering work of Larson \& Tinslev (1978), many studies have confirmed that tidal disturbance following galaxy interactions is an efficient trigger of star formation in galaxies (e.g. Joseph et al., 1984; Kennicutt \& Keel, 1984). Such star formation frequently takes the form of centrally-concentrated nuclear starbursts (Joseph \& Wright, 1985), fuelled by the central concentrations of molecular gas found to occur naturally in simulations of highly-disturbed systems (Barnes \& Hernquist, 1991; Mihos \& Hernquist, 1996). The strength of the link between starbursts and interactions was highlighted by the finding that almost all of the "ultra-luminous infrared galaxies' (ULIRGs) display signs of interactions or mergers (Sanders et al., 1988; Borne et al., 1999), and by correlations between galaxy-galaxy separations and starburst strength (Barton et al., 2000). Even minor mergers with low-mass companions have been shown through simulations to result in significant nuclear star formation activity (Mihos \& Hernquist, 1994).

Several early studies of nuclear starbursts suggested that this star formation might require a top heavy initial mass function (IMF), preferentially producing high mass stars (Rieke et al., 1980; Doyon et al., 1992). There is theoretical support for this suggestion, with simulations showing that an IMF weighted to high-mass stars naturally arises in high-density regions, due to feedback processes heating the gas. In a recent study, Krumholz et al. (2010) have demonstrated that such regions should have a high-mass stellar fraction at least 1.7 times larger, and possibly much more, than lower density, more quiescent regions.

However, the observational evidence for this variation has to date proved controversial (see Bastian et al. 2010 for a recent review). Some studies have found indirect evidence for top-heavy IMFs with, for example, Rieke et al. (1993) concluding that the nearby starburst galaxy M82 requires an IMF biased to high mass stars to explain its emission line ratios and total luminosity. Similar techniques have been used for NGC 3256 (an ongoing merger with a 'super-starburst') which have again shown indications of a modified IMF with an excess of high mass stars (Doyon et al., 1994). Gibson \& Matteucci (1997) showed that, in order to reproduce the observed colourluminosity relation of elliptical galaxies, an IMF much flatter than that of Salpeter (1955) needed to be adopted. Baugh et al. (2005) had to employ a top heavy IMF for the starbursts powering the distant population of highly luminous submillimetre galaxies in order to explain the number counts of these systems. Finally, Brassington et al. (2007) studied nine interacting galaxies from the Chandra survey and found that highly disturbed systems showed a strongly enhanced infrared luminosity compared to that expected from the x-ray emission, again suggesting the need for a top-heavy IMF.

More direct evidence of a variation in IMF has been found for the resolved stellar population of the young Arches cluster in the Galactic Centre. Figer et al. (1999); Stolte et al. (2002); Paumard et al. (2006); Espinoza et al. (2009) all find evidence for stellar mass functions weighted towards high-mass stars in this cluster or the general 
Galactic Centre region. Such mass functions are parametrized as an IMF that is either much flatter than that found by Salpeter (1955), or having a higher mass turnover than is found in the function for field stars.

One possible tracer of the IMF that has not been fully exploited to date is the relative numbers of core-collapse supernovae (CCSNe) of different types. Their short progenitor lifetimes and high luminosities make them powerful indicators of recent or ongoing star formation, and indeed they provide the only direct tracer of recent star formation within unresolved stellar populations. Recent advances in the understanding of supernovae and their progenitors raise the possibility that they can provide information on the initial mass function of a young stellar population. Theoretical models of single star progenitors predict that SNII should have lower

mass progenitors than SNIb or SNIc (Heger et al., 2003; Eldridge \& Tout, 2004). This has received observational support from studies of the strength of association with $\mathrm{H} \alpha$ emission (Anderson \& James, 2008), confirming that SNII have the lowest mass CC progenitors, but additionally indicating that the SNIc have still higher mass progenitors than the SNIb. The existence of this II-Ib-Ic progenitor mass sequence allows information on the IMF of the stellar population in the SN environments to be derived from the relative numbers of type II, Ib and Ic supernovae.

Petrosian \& Turatto (1995) studied the distribution of SNe events in 32 interacting systems containing 12 known core collapse SNe. They found that the radial distribution of these core collapse events showed a higher concentration towards the nuclear regions of the interacting galaxies when compared to isolated galaxies. This confirmed the enhanced star formation around the central regions of the systems, but the sample was too small to analyse the separate types of CCSNe.

This paper will therefore use a larger sample of local CCSNe to explore the IMF in nuclear starbursts, resulting from galaxy disturbance, by studying the ratio of type II/Ibc SNe in both disturbed and undisturbed host galaxies. Throughout this paper, we use 'Ibc' to encompass all SNe with classifications of Ib, Ic or Ib/c.

The structure of the paper is as follows: In Section 2 we will define and discuss the sample used throughout this work. Section 3 will describe the results on the radial distributions, for disturbed and undisturbed hosts and looking separately at type II and Ibc SNe. In Section 4 we discuss the possible interpretations of our results, in terms of metallicity, extinction and IMF effects. Finally, Section 5 contains a summary of our conclusions.

\section{Sample and observations}

The sample used in this work consists of 140 local (recession velocity $<6000 \mathrm{~km} / \mathrm{s}$ ) spiral galaxies, hosts to 178 CCSNe (110 SNII and 68 SNIbc), for which we have H $\alpha$ and $R$-band observations from the Liverpool Telescope (LT) and Isaac Newton Telescope (INT). (Some galaxies do not have usable images in either $\mathrm{H} \alpha$ or $R$-band and have been omitted from the corresponding plots and statistics; see Tables 1 \& 2 in the online 
material). This is the same dataset as was used by Anderson \& James (2009) with a small number of subsequent observations. SNe classified as type IIb are not included in this sample as they are thought to be transitional objects between SNII and SNIb, with substantially larger progenitor masses $\left(\sim 25 \mathrm{M}_{\odot}\right.$; Smartt 2009$)$ than typical SNII. A comparison performed on January 21st 2010 with all CCSNe host galaxies within the same recession velocity limit in the IAU SN cataloguet and where the SNe have accurate classifications and positions, showed this sample to be $\sim 34 \%$ complete for SNIbc and $\sim 18 \%$ complete for SNII.

The classification of host galaxies as disturbed is purely by visual inspection by the authors and thus is subjective. Galaxies which show signs of tidal tails, definite interaction, double nuclei or strong asymmetry have therefore been classed as disturbed.

\section{Results}

The total sample of CCSNe is dominated by SNII (62\% of the total). When the sample is constrained only to supernovae which lie in disturbed hosts (64 CCSNe) this falls to $56 \%$ SNII, compared to $65 \%$ SNII in the non-disturbed hosts.

For each of the CCSNe in our sample we have calculated the $\operatorname{Fr}(R)$ and $\operatorname{Fr}(\mathrm{H} \alpha)$ statistics used, and explained fully, in Anderson \& James (2009). Briefly, these represent the fractions of galaxy emission, in the $R$-band and $\mathrm{H} \alpha$ respectively, that lie within the circle or ellipse which contains the SN. Thus $\operatorname{Fr}(R)=0.0$ corresponds to a supernova at the central $R$-band peak of the galaxy emission, or closer to this peak than any $\mathrm{H} \alpha$ emission, in the case of $\operatorname{Fr}(\mathrm{H} \alpha)$; whilst $\mathrm{Fr}=1.0$ implies an extreme outlying $\mathrm{SN}$. If the emission is statistically a good tracer of the parent population of supernovae, the Fr values should have a flat distribution with a mean value of 0.5 .

Figures 1 and 2 show the distributions of $\operatorname{Fr}(R)$ values for the CCSNe in the present sample, for the undisturbed and disturbed galaxies respectively. In all histograms shown in this paper, the upper plot represents the CCSNe sample, the middle the type II SNe and the lower SNIbc. Looking first at the overall distributions of CCSNe, there is a clear difference between the disturbed and undisturbed subsets, in the sense that the disturbed galaxies have substantially more CCSNe occurring in their central regions, with low $\operatorname{Fr}(R)$ values. For example, 36 of the 58 CCSNe in the disturbed sample occur within the central $50 \%$ of the $R$-band light, $62 \%$ of the total, compared with 50 out of $112(45 \%)$ in the undisturbed galaxies. A Kolmogorov-Smirnov (KS) test shows that the chance of the two total CCSNe distributions being drawn from the same parent distribution is $\mathrm{P}=0.037$. Thus there is evidence at the $2 \sigma$ level that galaxy disturbance correlates with centrally-enhanced star formation and hence the production of an increased central fraction of CCSNe.

The most striking aspect of Figure 2 is the types of SNe that make up this central excess in the disturbed galaxies. Remarkably, given that SNIbc only comprise $38 \%$ of the overall CCSN sample (68/178), all 5 of the CCSNe coming from the central $10 \%$ of

$\ddagger$ http://www.cfa.harvard.edu/iau/lists/Supernovae.html 
the disturbed host galaxy light, and 11 of the 13 coming from the central $20 \%$ of the light, are of type Ibc. A KS test of the $\operatorname{Fr}(R)$ distributions for the disturbed galaxy subsample finds $\mathrm{P}=0.003$, indicating a very low probability that the SNIbc and SNII $\operatorname{Fr}(R)$ values are drawn from the same parent distribution. The mean values of $\operatorname{Fr}(R)$ are 0.31 (95\% confidence limits $0.20-0.42)$ for the SNIbc in the disturbed galaxies, compared with $0.51(0.44-0.59)$ for the SNII in the disturbed galaxies. This is the main observational result from this paper; the CCSNe occurring in the central regions of disturbed galaxies are heavily weighted towards types Ib, Ic and Ib/c. We will discuss possible interpretations of this in Section 4.

Some further statistical tests were also performed on the CCSN distributions shown in Figures $1 \& 2$. Figure 1 shows that even in the undisturbed galaxies, there is some evidence for a larger fraction of SNIbc in the central regions, principally due to a central 'hole' in the radial distribution of SNII. A KS test applied to the SNIbc and SNII distributions shown in Figure 1 shows this difference to be only marginal, $\mathrm{P}=0.082$, and hence clearly less marked than for the disturbed galaxies; disturbance does seem to play a part in the central concentration of the SNIbc. This point was further explored by comparing the SNIbc distributions for undisturbed and disturbed galaxies, i.e. Figure 1 vs. Figure 2; this did indicate the SNIbc in disturbed galaxies to be more centrally concentrated, with a KS P value of 0.06 , again of marginal significance. The mean SNIbc $\operatorname{Fr}(R)$ value is $0.48(0.38-0.57)$ for the SNIbc in the undisturbed galaxies, again to be compared with $0.31(0.20-0.42)$ already quoted for the disturbed galaxies. Finally for Figure 2, it might be asked whether there is evidence for a suppression of SNIbc fraction in the outer regions of these galaxies. However, given the current sample size this cannot be determined with any significance. For example we find 6 SNIbc in the outer $50 \%$ of the light distributions of the disturbed galaxies, but with only 22 CCSNe in total from these regions, this is not significantly below the expectation value of 8.4 , based on the SNIbc/SNII ratio for the full sample.

Figures $3 \& 4$ show the distributions of supernova locations relative to the $\mathrm{H} \alpha$ distributions of their host galaxies. Overall these show the same patterns as Figures 1 $\& 2$, but they do enable one specific issue to be addressed: are the SNIbc more centrally concentrated than the $\mathrm{H} \alpha$ light, which is presumably a good tracer of the youngest stellar population? Figure 4 shows that there is some evidence for this; the central $10 \%$ of the $\mathrm{H} \alpha$ emission in the disturbed galaxies gives rise to 7 of the 22 SNIbc in these galaxies. The mean $\operatorname{Fr}(\mathrm{H} \alpha)$ value for the SNIbc in disturbed galaxies is 0.33 (0.21-0.45), so this population does seem to be more centrally concentrated than the $\mathrm{H} \alpha$ emission. This is not true for the SNIbc in the undisturbed galaxies, or for the SNII in either of the galaxy subsets; all of these distributions have mean $\operatorname{Fr}(\mathrm{H} \alpha)$ values consistent with 0.5 .

\section{Discussion}

Anderson \& James (2009) found a central excess of SNIbc in a SN-host galaxy sample. 
This work has found that this central excess is exaggerated in galaxies which appear disturbed. A more centrally located distribution of SNIbc has been suggested previously (e.g. Bartunov et al., 1992; Petrosian \& Turatto, 1995; van den Bergh, 1997), though previous studies often suffered from low number statistics. Hakobyan et al. (2009) also find SNIbc to be more centrally located than SNII, however in conflict to our results they do not find the central excess of SNIbc clearly seen in our data. An important difference between our work and most other studies in the literature is that our method implicitly normalizes the tests to the measured distributions of different stellar populations; other studies use distances normalized to isophotal radii. Most of these results have been interpreted as an increase in metallicity of the SNIbc progenitors, although Hakobyan et al. (2009) also make the suggestion of a shallower IMF within the central regions.

Studies conducted into active and star-forming galaxies (Petrosian et al., 2005) and Seyfert galaxies (Bressan et al., 2002) have also noted marginal evidence for an increased fraction of both CCSNe and specifically SNIbc within these galaxies when compared to 'normal' ones.

There are various observational biases which may affect our analysis. Shaw (1979) found a bias in supernova samples, in the sense that it is more difficult to detect SNe in the inner regions of distant galaxies. The sample is also subject to any bias contained within the object selection found in the Asiago (Barbon et al., 2009) and IAU SN catalogues. For the Asiago and Crimea searches, Cappellaro et al. (1993) estimated the number of SNe lost due to overexposure combined with the Shaw effect, which for the velocity range of our sample is $\sim 35 \%$. Another source of bias is the loss of $\mathrm{SNe}$ in the central regions of galaxies through the large amount of dust obscuration which has been investigated through near infrared studies (e.g. Mattila et al., 2007; Kankare et al., 2008). Such biases should affect all SN types, although the intrinsically fainter SNIIP (Richardson et al., 2002, 2006) may be rather more likely to be lost through these effects. However, if our results are correct and SNIbc are more centrally concentrated than SNII then recovering all of the lost central SNe would lead to an even more exaggerated excess.

One possible source of error is our eyeball classifications of host galaxy disturbance. In future we plan to quantify this through near-IR observations and use of objective measures of asymmetry (Conselice et al., 2000; Lotz et al., 2004). However, we are quite confident in our disturbance classifications; images of 12 of our 'disturbed' galaxies with centrally-located SNe are shown in Figure 5, confirming that this is not a 'normal' group of galaxies.

The high central excess of SNIbc in the central regions of the disturbed host galaxies found in this work is difficult to explain in terms of effects other than an IMF biased towards high-mass stars. A possible alternative explanation is the effect of metallicity, given that Boissier \& Prantzos (2009) find that the ratio of SNIbc/SNII increases with both local and global metallicity. Looking at the absolute magnitudes of the host galaxies, we do indeed find that the disturbed galaxies are somewhat more luminous 
than the undisturbed galaxies (KS probability of 0.07 that they are drawn from the same parent distribution), by almost 0.4 mag in the mean, which might imply a somewhat higher mean metallicity in the disturbed galaxies. However, this does not seem to be driving the result we find. Splitting the total sample (disturbed and undisturbed) by absolute magnitude, we find no significant differences in the $\operatorname{Fr}(R)$ distributions of bright and faint galaxies. Splitting into bright and faint halves, the KS probability is 0.998 (complete consistency), whereas when the bright third is compared with the faintest two-thirds (to better match the disturbed/undisturbed split), $\mathrm{P}$ is 0.276 , but in the sense that the bright galaxies have a slight bias towards high $\operatorname{Fr}(R)$ values. In any case, the expected metallicity bias resulting from a difference of 0.4 in galaxy absolute magnitude is very small. The mass-metallicity relation of Tremonti et al. (2004) for galaxies of a few times $10^{10} \mathrm{M}_{\odot}$ predicts a corresponding change of only $\sim 0.025$ dex in $\log (\mathrm{O} / \mathrm{H})$, highly unlikely to cause any significant effects. Finally, in interacting systems the central metallicity is lowered by the in-fall of unenriched gas (Michel-Dansac et al., 2008; Ellison et al., 2008; Rupke et al., 2010). This would therefore act in the opposite sense to the result we find. It should also be noted that whilst a study of gas-phase metallicities of the local environments of CCSNe (Anderson et al. MNRAS submitted) finds a trend favouring SNIbc in high-metallicity regions, even the highest metallicity environments seem to host a significant fraction of SNII.

It is also possible that stellar rotation (e.g. Heger et al., 2003) and binarity (e.g. Nomoto et al., 1995) could contribute to this effect. It is not clear why the binary fraction should be higher within the disturbed galaxy sample, but it should be noted that the increased densities within these nuclear starburst regions could lead to more massive and denser clusters, within which processes such as stellar mergers and binary interactions would be more prevalent (e.g. Portegies Zwart et al., 2010).

To conclude, our preferred explanation of this central excess of SNIbc is that the central regions of these disturbed galaxies are hosting starbursts with initial mass functions biased to high stellar masses. Given the small numbers of SNe involved, the uncertain mass limits corresponding to progenitors of different SN types, and the likely role of binarity in determining SN type, it is hard to quantify the implications of this result for the IMF. However, an illustrative calculation can be performed as follows. Under the assumption of a Salpeter IMF, and the (admittedly simplistic) assumptions that CCSNe arise from single stars with masses between 8 and $80 \mathrm{M}_{\odot}$ and that mass alone determines SN type, the relative numbers of SNII and Ibc in the outer regions of undisturbed galaxies (2.3:1) indicate a transition at about $18 \mathrm{M}_{\odot}$. The apparent inversion of this ratio for the central regions of the disturbed galaxies $(0.18: 1)$, if interpreted purely as a change in IMF slope, appears to require a positive index in the IMF slope (formally $x=+0.95 \mathrm{cf}$. -1.35 , assuming the transition mass is unchanged at $\left.18 \mathrm{M}_{\odot}\right)$. However, we emphasize that this is purely illustrative; all of the assumptions are likely to be in error at some level, and binarity and metallicity effects may play some part in the changes we find.

We note here that we are modifying the conclusions of Anderson \& James (2009) in 
that it is hard to interpret the previously found central SNIbc excess purely in terms of metallicity effects. However, we note that there is still a marginal central excess of SNIbc in the undisturbed galaxy sample, indicating some effect of metallicity. Quantifying the relative sizes of the different effects will be the focus of future studies.

Finally, it is interesting to note that with current research indicating a connection between gamma ray bursts (GRBs) and type Ic SNe (Wooslev \& Bloom, 2006), recent studies (e.g. Conselice et al., 2005; Wainwright, 2007; Fryer et al., 2007) have found that GRB host galaxies show an over-abundance of merging or interacting galaxies compared to other star-forming hosts.

\section{Conclusions}

We have analysed the spatial distribution of 178 CCSNe within a sample of host galaxies with recession velocities less than $6000 \mathrm{~km} / \mathrm{s}$. Host galaxies were classified by eye according to whether they show disturbance due to strong tidal interactions or mergers. The main results are as follows:

- CCSNe of all types show a strong degree of central concentration in the disturbed galaxies, probably as a result of nuclear starbursts in these galaxies.

- This central excess is dominated by SNIbc.

- The SNIbc in disturbed galaxies are more centrally concentrated than the $\mathrm{H} \alpha$ emission.

- The SNIbc excess cannot easily be explained in terms of metallicity effects, extinction, or central incompleteness of SNe.

- Our preferred explanation of the SNIbc excess is that the central regions of the disturbed galaxies are dominated by nuclear starbursts with IMFs biased towards high mass stars, although metallicity, binarity and stellar rotation may also play a role.

\section{Acknowledgments}

This paper has made use of data provided by the Central Bureau for Astronomical Telegrams. We would like to acknowledge members of staff at the Astrophysics Research Institute, in particular Sue Percival and David Bersier for their helpful comments and discussion. The authors would also like to thank the referee for a constructive and helpful report. This research has made use of the NASA/IPAC Extragalactic Database (NED) which is operated by the Jet Propulsion Laboratory, California Institute of Technology, under contract with the National Aeronautics and Space Administration. The Isaac Newton Telescope is operated on the island of La Palma by the Isaac Newton Group in the Spanish Observatorio del Roque de los Muchachos of the Instituto de Astrofísica de Canarias. The Liverpool Telescope is operated on the island of La Palma by Liverpool John Moores University in the Spanish Observatorio del Roque de los Muchachos of the 
Instituto de Astrofísica de Canarias with financial support from the UK Science and Technology Facilities Council. SMH would like to acknowledge STFC for a research studentship. 


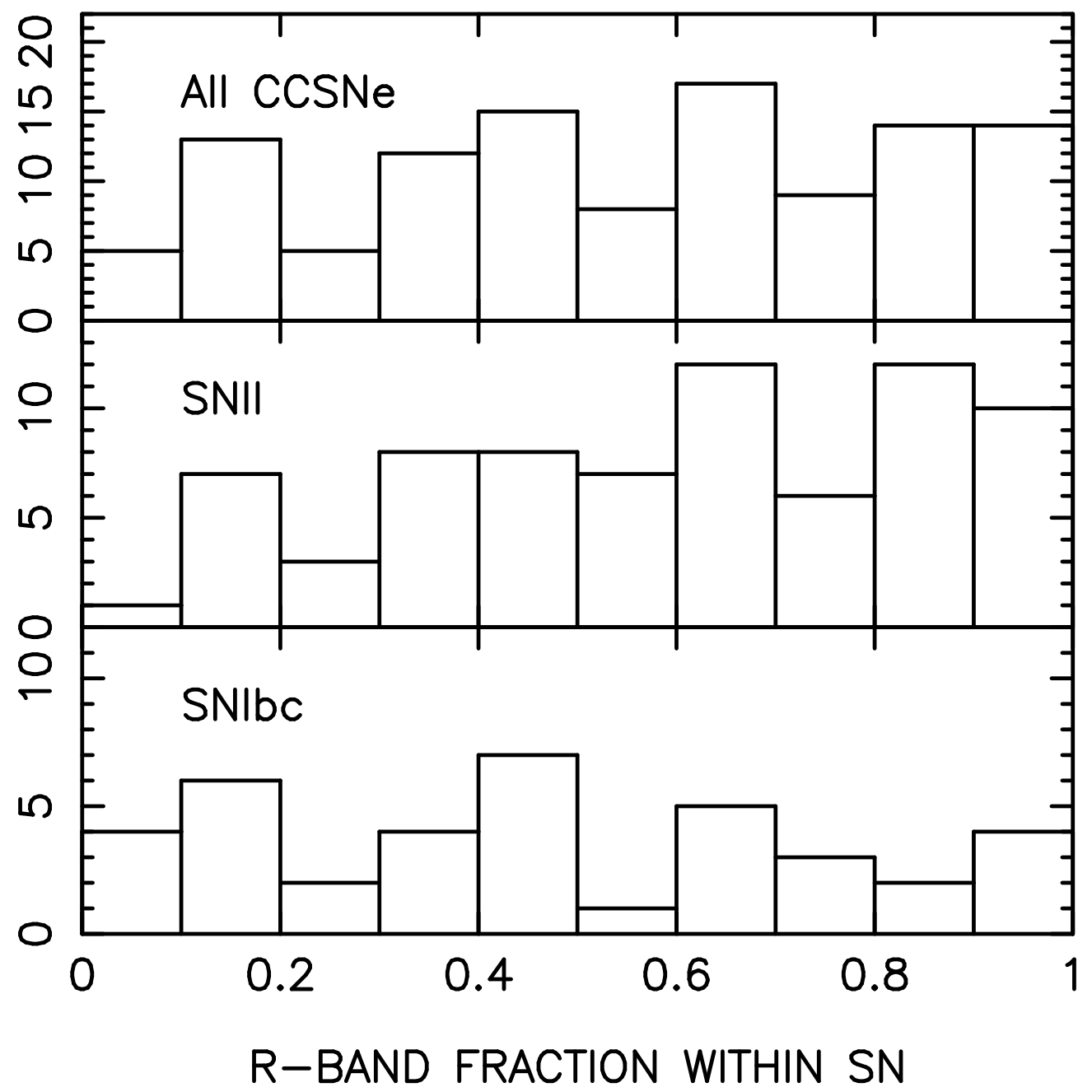

Figure 1. Histogram showing the distribution of fractions of host galaxy $R$-band light lying within the locations of each CCSN in our undisturbed host galaxies. The top plot represents the distribution of all CCSNe, the middle SNII and the lower SNIbc. 


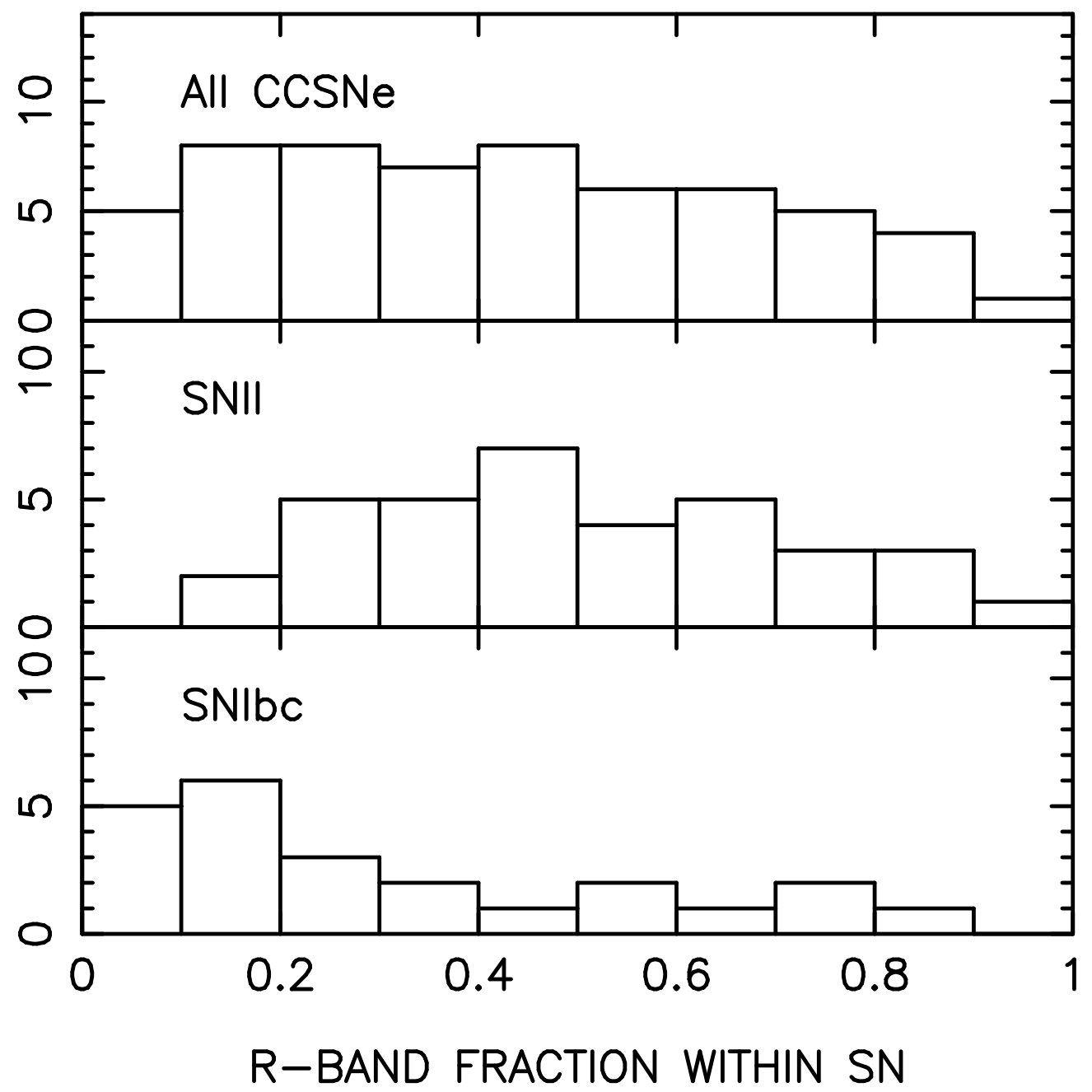

Figure 2. As Figure 1, but for the disturbed host galaxies. 


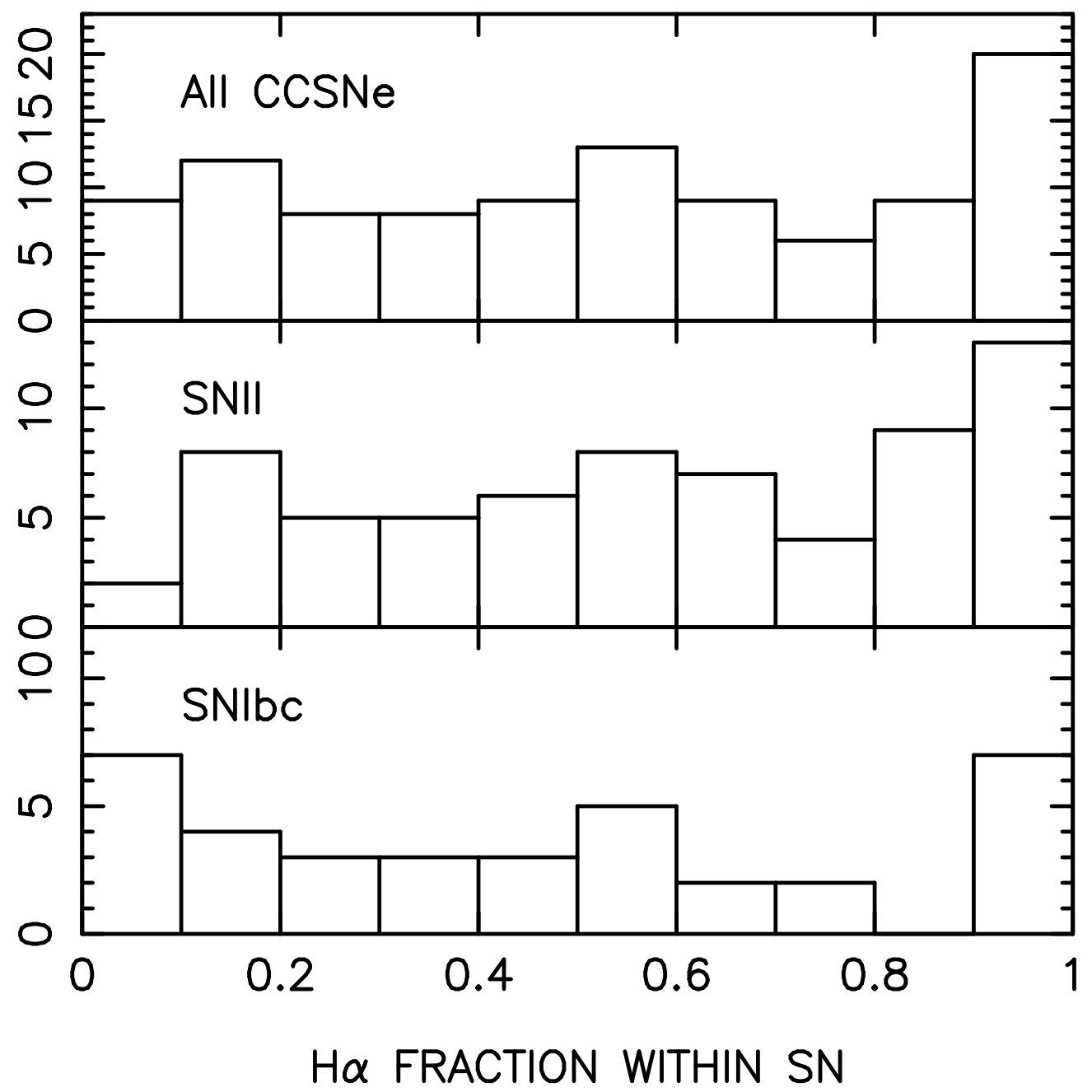

Figure 3. Histogram showing the distribution of fractions of host galaxy $\mathrm{H} \alpha$ light lying within the locations of each CCSN in our sample, for the undisturbed host galaxies. Again, the upper plot shows the overall CCSNe distribution, the middle SNII and the lower SNIbc. 


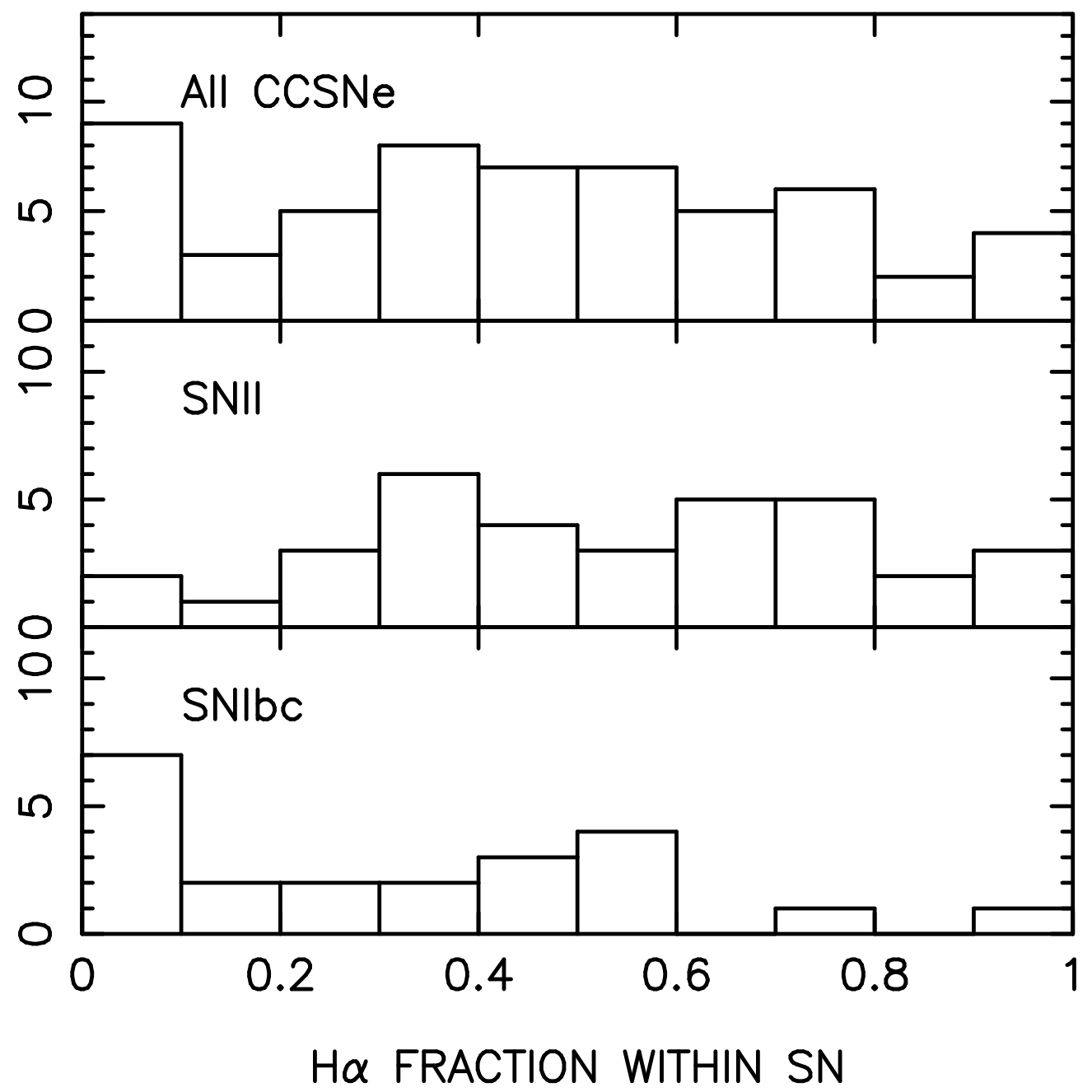

Figure 4. As Figure 3, for the disturbed host galaxies. 


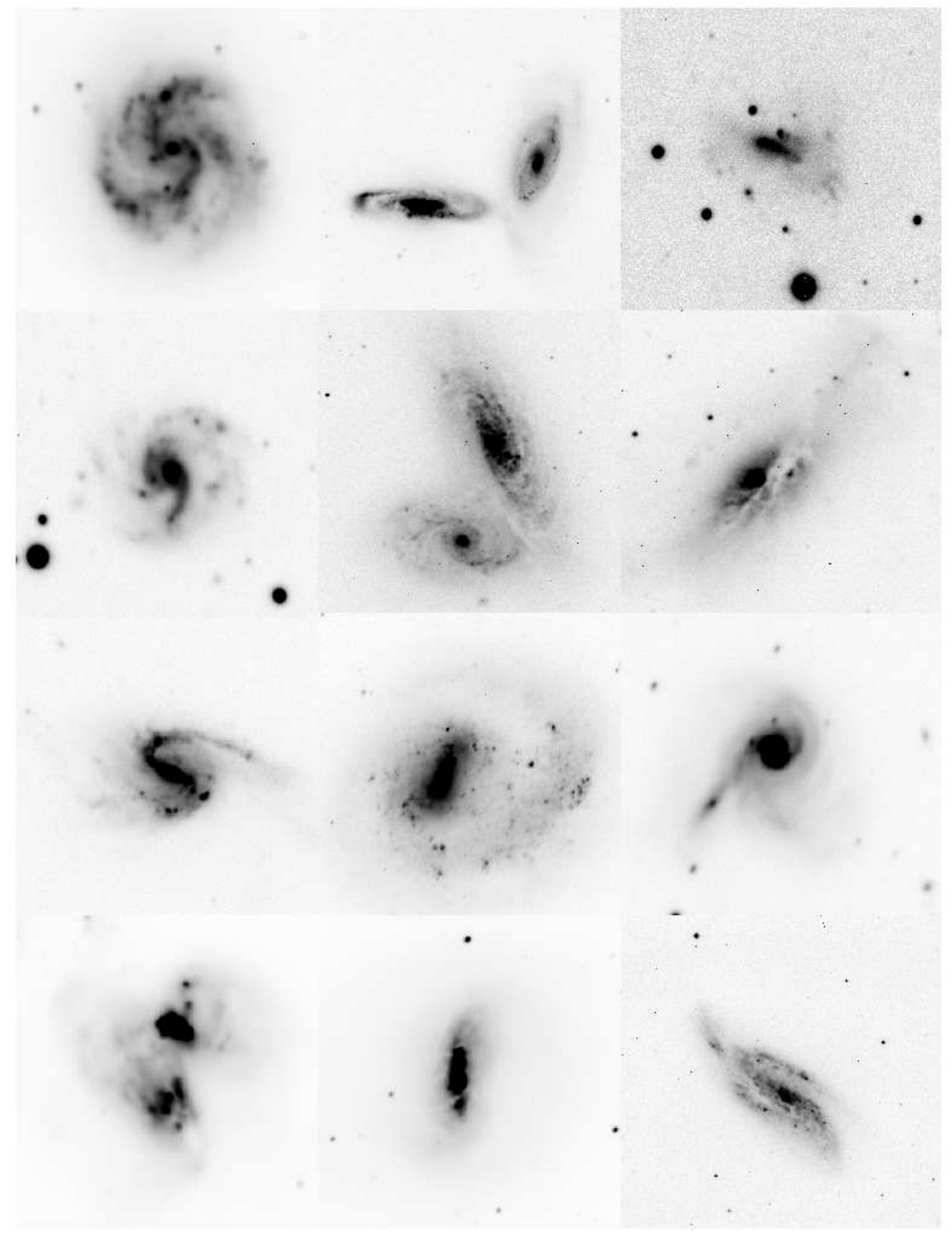

Figure 5. Images of 12 of the host galaxies classified as disturbed and with centrally located CCSNe. 
Supernovae and the IMF in disturbed galaxies

Tables and table captions

Table 1: Undisturbed host galaxy sample used in this analysis. Columns represent the host galaxy, the individual SNe, the spectral classification of the $\mathrm{SNe}$ and the fractional $R$-band light and fractional $\mathrm{H} \alpha$ values for each SNe.

\begin{tabular}{|c|c|c|c|c|}
\hline Host & $\mathrm{SN}$ & SN type & $\operatorname{Fr}(R)$ & $\operatorname{Fr}(\mathrm{H} \alpha)$ \\
\hline NGC493 & $1971 \mathrm{~S}$ & IIP & 0.605 & 0.570 \\
\hline NGC918 & $2009 \mathrm{js}$ & IIP & 0.703 & - \\
\hline NGC941 & 2005ad & II & 0.831 & 0.864 \\
\hline NGC991 & $1984 \mathrm{~L}$ & $\mathrm{Ib}$ & 0.498 & 0.401 \\
\hline NGC1035 & $1990 \mathrm{E}$ & IIP & 0.272 & 0.363 \\
\hline NGC1058 & $1961 \mathrm{~V}$ & II & 0.968 & 0.931 \\
\hline NGC1058 & 1969L & IIP & 1.000 & 1.000 \\
\hline NGC1058 & $2007 \mathrm{gr}$ & $\mathrm{Ib} / \mathrm{c}$ & 0.421 & - \\
\hline NGC1073 & $1962 \mathrm{~L}$ & Ic & 0.754 & 0.518 \\
\hline NGC1087 & $1995 \mathrm{~V}$ & II & 0.368 & 0.497 \\
\hline NGC1187 & $1982 R$ & $\mathrm{Ib}$ & 0.695 & 0.760 \\
\hline NGC1187 & $2007 \mathrm{Y}$ & $\mathrm{Ib}$ & 0.981 & 1.000 \\
\hline MCG-01-09-24 & 2002ei & IIP & 0.195 & 0.195 \\
\hline NGC1343 & $2008 \mathrm{dv}$ & Ic & 0.195 & 0.134 \\
\hline UGC2906 & 2008im & $\mathrm{Ib}$ & 0.682 & - \\
\hline UGC2971 & 2003ig & Ic & 0.176 & 0.108 \\
\hline IC381 & $2001 \mathrm{ef}$ & Ic & 0.082 & 0.052 \\
\hline NGC1832 & $2004 \mathrm{gq}$ & $\mathrm{Ib}$ & 0.672 & 0.328 \\
\hline NGC1832 & $2009 \mathrm{kr}$ & II & 0.489 & - \\
\hline IC2152 & $2004 \mathrm{ep}$ & II & 0.461 & 0.560 \\
\hline UGC3804 & $2002 \mathrm{~A}$ & IIn & 0.419 & 0.253 \\
\hline NGC2551 & $2003 \mathrm{hr}$ & II & 0.914 & 1.000 \\
\hline NGC2596 & 2003bp & $\mathrm{Ib}$ & 0.486 & 0.362 \\
\hline UGC4436 & $2004 \mathrm{ak}$ & II & 0.887 & 0.882 \\
\hline NGC2726 & $1995 \mathrm{~F}$ & Ic & 0.037 & 0.050 \\
\hline NGC2742 & $2003 Z$ & IIP & 0.675 & 0.736 \\
\hline NGC2715 & $1987 \mathrm{M}$ & Ic & 0.129 & 0.044 \\
\hline UGC4904 & $2006 \mathrm{jc}$ & $\mathrm{Ib} / \mathrm{c}$ & 0.332 & 0.525 \\
\hline NGC2841 & $1972 \mathrm{R}$ & $\mathrm{Ib}$ & 0.855 & 0.904 \\
\hline NGC2906 & 2005ip & II & 0.399 & 0.528 \\
\hline UGC5249 & $1989 \mathrm{C}$ & IIP & 0.017 & 0.058 \\
\hline NGC3074 & $1965 \mathrm{~N}$ & IIP & 0.110 & 0.059 \\
\hline NGC3074 & $2002 \mathrm{cp}$ & $\mathrm{Ib} / \mathrm{c}$ & 0.936 & 0.961 \\
\hline NGC3147 & 2006gi & $\mathrm{Ib}$ & 0.984 & 0.991 \\
\hline NGC3184 & 1921B & II & 0.856 & 0.954 \\
\hline NGC3184 & $1937 \mathrm{~F}$ & IIP & 0.808 & 0.930 \\
\hline NGC3184 & 1999gi & IIP & 0.276 & 0.112 \\
\hline NGC3198 & 1966J & $\mathrm{Ib}$ & 0.898 & 0.963 \\
\hline NGC3198 & $1999 \mathrm{bw}$ & IIn & 0.745 & 0.755 \\
\hline NGC3240 & $2001 \mathrm{M}$ & Ic & 0.323 & 0.251 \\
\hline NGC3294 & $1990 \mathrm{H}$ & IIP & 0.156 & 0.125 \\
\hline NGC3340 & $2005 \mathrm{O}$ & $\mathrm{Ib}$ & 0.322 & 0.305 \\
\hline
\end{tabular}




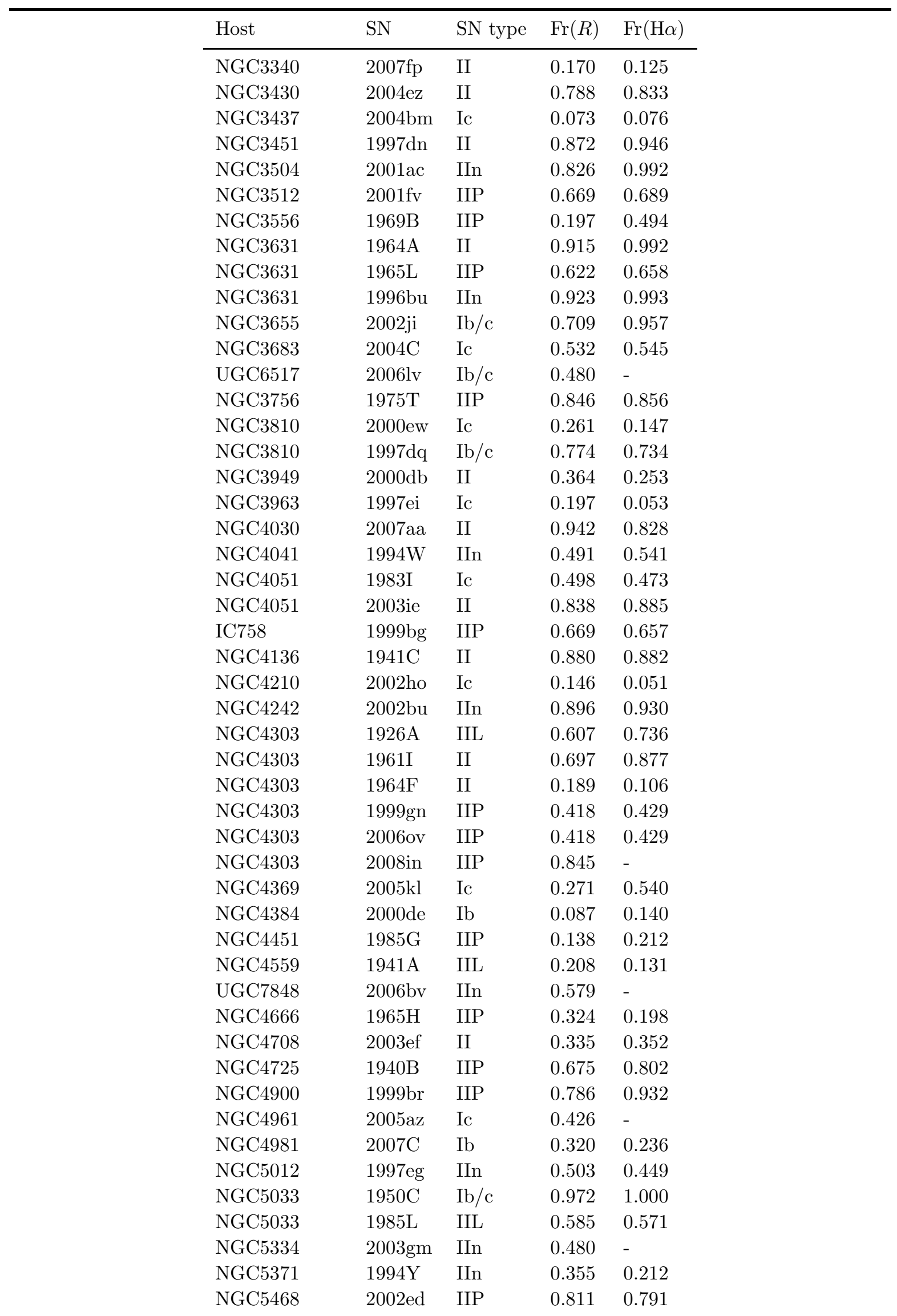




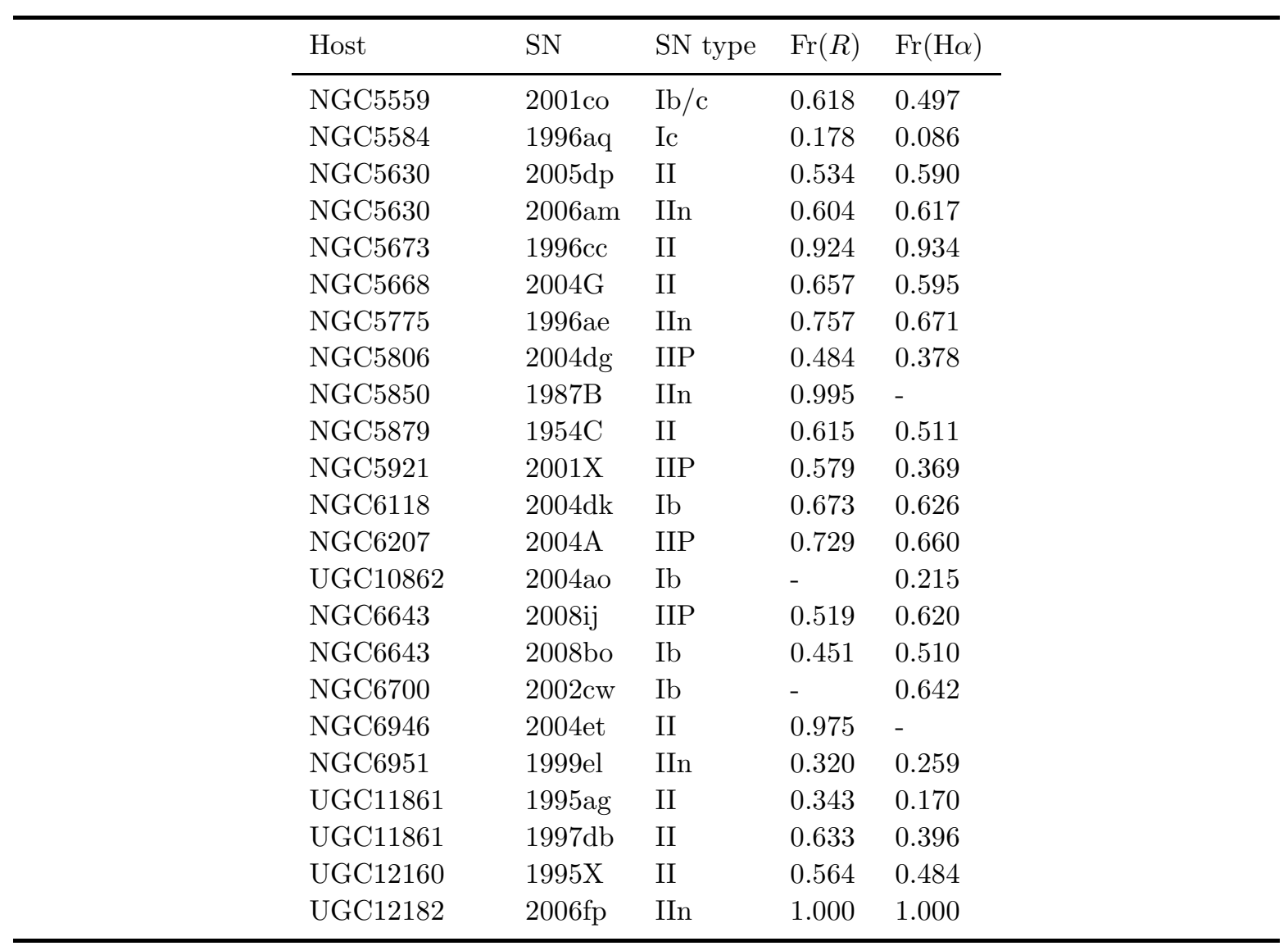


Supernovae and the IMF in disturbed galaxies

Table 2: Disturbed host galaxy sample used in this analysis. Columns represent the host galaxy, the individual SNe, the spectral classification of the $\mathrm{SNe}$ and the fractional $R$-band light and fractional $\mathrm{H} \alpha$ values for each $\mathrm{SNe}$, as in table 1.

\begin{tabular}{|c|c|c|c|c|}
\hline Host & $\mathrm{SN}$ & SN type & $\operatorname{Fr}(R)$ & $\operatorname{Fr}(\mathrm{H} \alpha)$ \\
\hline NGC895 & 2003id & Ic & 0.524 & - \\
\hline UGC2984 & $2002 \mathrm{jz}$ & Ic & 0.091 & 0.099 \\
\hline NGC1614 & 1996D & Ic & 0.275 & - \\
\hline NGC1637 & $1999 \mathrm{em}$ & IIP & 0.276 & 0.268 \\
\hline IC391 & 2001B & $\mathrm{Ib}$ & 0.062 & 0.060 \\
\hline NGC1961 & 2001 is & $\mathrm{Ib}$ & - & 0.749 \\
\hline NGC2207 & $1999 \mathrm{ec}$ & $\mathrm{Ib}$ & - & 0.521 \\
\hline NGC2207 & $2003 \mathrm{H}$ & $\mathrm{Ib}$ & - & 0.259 \\
\hline NGC2146 & $2005 \mathrm{~V}$ & $\mathrm{Ib} / \mathrm{c}$ & 0.033 & 0.091 \\
\hline ESO492-G2 & $2005 \mathrm{lr}$ & Ic & - & 0.005 \\
\hline UGC3829 & 2001ej & $\mathrm{Ib}$ & 0.152 & 0.391 \\
\hline NGC2276 & $1968 \mathrm{~V}$ & II & 0.699 & 0.790 \\
\hline NGC2276 & $2005 \mathrm{dl}$ & II & 0.247 & 0.099 \\
\hline NGC2276 & 1993X & II & 0.899 & 0.619 \\
\hline NGC2532 & $1999 \mathrm{gb}$ & IIn & 0.485 & 0.443 \\
\hline NGC2532 & $2002 \mathrm{hn}$ & Ic & 0.023 & 0.011 \\
\hline NGC2604 & 2002ce & II & 0.381 & 0.560 \\
\hline NGC2782 & 1994ak & IIn & 0.725 & 0.977 \\
\hline NGC2993 & $2003 \mathrm{ao}$ & IIP & 0.456 & 0.784 \\
\hline NGC3169 & $1984 \mathrm{E}$ & IIL & 0.684 & 0.731 \\
\hline NGC3310 & $1991 \mathrm{~N}$ & Ic & 0.268 & 0.277 \\
\hline NGC3323 & $2004 \mathrm{bs}$ & $\mathrm{Ib}$ & 0.191 & 0.119 \\
\hline NGC3323 & $2005 \mathrm{kk}$ & II & 0.766 & 0.875 \\
\hline NGC3367 & $1992 \mathrm{C}$ & II & 0.689 & 0.687 \\
\hline NGC3367 & $2007 \mathrm{am}$ & II & 0.302 & 0.314 \\
\hline NGC3627 & 1973R & IIP & 0.471 & 0.566 \\
\hline NGC3627 & $1997 \mathrm{bs}$ & IIn & 0.362 & 0.348 \\
\hline NGC3627 & 2009hd & II & 0.496 & - \\
\hline NGC3690 & $1993 \mathrm{G}$ & IIL & 0.464 & 0.744 \\
\hline NGC3690 & $1998 \mathrm{~T}$ & $\mathrm{Ib}$ & 0.056 & 0.056 \\
\hline NGC3690 & 1999D & II & 0.560 & 0.849 \\
\hline NGC3786 & 1999bu & Ic & 0.180 & 0.522 \\
\hline NGC3811 & $1971 \mathrm{~K}$ & IIP & 0.809 & 0.900 \\
\hline IC2973 & $1991 \mathrm{~A}$ & Ic & 0.742 & 0.588 \\
\hline NGC4038 & $2004 \mathrm{gt}$ & $\mathrm{Ib} / \mathrm{c}$ & 0.834 & 0.991 \\
\hline NGC4088 & $1991 \mathrm{G}$ & IIP & 0.466 & 0.453 \\
\hline NGC4088 & $2009 \mathrm{dd}$ & II & 0.100 & - \\
\hline NGC4141 & $2008 X$ & IIP & 0.194 & 0.085 \\
\hline NGC4141 & $2009 \mathrm{E}$ & IIP & 0.594 & 0.491 \\
\hline NGC4254 & $1967 \mathrm{H}$ & II & 0.664 & 0.648 \\
\hline NGC4254 & $1972 Q$ & IIP & 0.811 & 0.791 \\
\hline NGC4254 & $1986 \mathrm{I}$ & IIP & 0.334 & 0.318 \\
\hline NGC4273 & $2008 \mathrm{~N}$ & IIP & 0.300 & 0.316 \\
\hline NGC4273 & $1936 \mathrm{~A}$ & IIP & 0.569 & 0.598 \\
\hline NGC4490 & $1982 \mathrm{~F}$ & IIP & 0.277 & 0.202 \\
\hline
\end{tabular}




\begin{tabular}{llllll}
\hline Host & SN & SN type & $\operatorname{Fr}(R)$ & $\operatorname{Fr}(\mathrm{H} \alpha)$ \\
\cline { 2 - 5 } NGC4568 & $1990 \mathrm{~B}$ & Ic & 0.302 & - \\
NGC4568 & 2004cc & Ic & 0.158 & - \\
NGC4618 & $1985 \mathrm{~F}$ & Ib & 0.121 & 0.087 \\
NGC4615 & $1987 \mathrm{~F}$ & IIn & 0.489 & 0.333 \\
NGC4688 & $1966 \mathrm{~B}$ & IIL & 0.571 & 0.454 \\
NGC4691 & $1997 \mathrm{X}$ & Ic & 0.171 & 0.472 \\
NGC5000 & 2003el & Ic & 0.482 & 0.476 \\
NGC5021 & $1996 \mathrm{ak}$ & II & 0.619 & 0.659 \\
MCG-04-32-07 & 2003am & II & 0.211 & 0.339 \\
NGC5194 & 1994I & Ic & - & 0.122 \\
NGC5194 & 2005cs & IIP & - & 0.222 \\
NGC5395 & 2000cr & Ic & 0.538 & 0.549 \\
NGC5480 & 1988L & Ib & 0.230 & 0.369 \\
NGC5682 & 2005ci & II & 0.204 & 0.191 \\
NGC7479 & 1990U & Ic & 0.603 & 0.488 \\
NGC7479 & 2009jf & Ib & 0.764 & - \\
NGC7537 & 2002gd & II & 0.759 & 0.685 \\
NGC7714 & 2007fo & Ib & 0.377 & - \\
UGC12846 & 2007od & IIP & 0.945 & 1.000 \\
\hline
\end{tabular}




\section{References}

Anderson, J. P. \& James, P. A. 2008, MNRAS, 390, 1527

—. 2009, MNRAS, 399, 559

Barbon, R., Buondi, V., Cappellaro, E., \& Turatto, M. 2009, VizieR Online Data Catalog, 1, 2024

Barnes, J. E. \& Hernquist, L. E. 1991, ApJ, 370, L65

Barton, E. J., Geller, M. J., \& Kenyon, S. J. 2000, ApJ, 530, 660

Bartunov, O. S., Makarova, I. N., \& Tsvetkov, D. I. 1992, A\&A, 264, 428

Bastian, N., Covey, K. R., \& Meyer, M. R. 2010, ArXiv e-prints

Baugh, C. M., Lacey, C. G., Frenk, C. S., Granato, G. L., Silva, L., Bressan, A., Benson, A. J., \& Cole, S. 2005, MNRAS, 356, 1191

Boissier, S. \& Prantzos, N. 2009, A\&A, 503, 137

Borne, K. D., Bushouse, H., Colina, L., Lucas, R. A., Baker, A., Clements, D., Lawrence, A., Oliver, S., \& Rowan-Robinson, M. 1999, Ap\&SS, 266, 137

Brassington, N. J., Ponman, T. J., \& Read, A. M. 2007, MNRAS, 377, 1439

Bressan, A., Della Valle, M., \& Marziani, P. 2002, MNRAS, 331, L25

Cappellaro, E., Turatto, M., Benetti, S., Tsvetkov, D. Y., Bartunov, O. S., \& Makarova, I. N. 1993, A\&A, 273, 383

Conselice, C. J., Bershady, M. A., \& Jangren, A. 2000, ApJ, 529, 886

Conselice, C. J., Vreeswijk, P. M., Fruchter, A. S., Levan, A., Kouveliotou, C., Fynbo, J. P. U., Gorosabel, J., Tanvir, N. R., \& Thorsett, S. E. 2005, ApJ, 633, 29

Doyon, R., Joseph, R. D., \& Wright, G. S. 1994, ApJ, 421, 101

Doyon, R., Puxley, P. J., \& Joseph, R. D. 1992, ApJ, 397, 117

Eldridge, J. J. \& Tout, C. A. 2004, MNRAS, 353, 87

Ellison, S. L., Patton, D. R., Simard, L., \& McConnachie, A. W. 2008, AJ, 135, 1877

Espinoza, P., Selman, F. J., \& Melnick, J. 2009, A\&A, 501, 563

Figer, D. F., Kim, S. S., Morris, M., Serabyn, E., Rich, R. M., \& McLean, I. S. 1999, ApJ, 525, 750

Fryer, C. L., Mazzali, P. A., Prochaska, J., Cappellaro, E., Panaitescu, A., Berger, E., van Putten, M., van den Heuvel, E. P. J., Young, P., Hungerford, A., Rockefeller, G., Yoon, S., Podsiadlowski, P., Nomoto, K., Chevalier, R., Schmidt, B., \& Kulkarni, S. 2007, PASP, 119, 1211

Gibson, B. K. \& Matteucci, F. 1997, MNRAS, 291, L8

Hakobyan, A. A., Mamon, G. A., Petrosian, A. R., Kunth, D., \& Turatto, M. 2009, A\&A, 508, 1259

Heger, A., Fryer, C. L., Woosley, S. E., Langer, N., \& Hartmann, D. H. 2003, ApJ, 591, 288 
Joseph, R. D., Meikle, W. P. S., Robertson, N. A., \& Wright, G. S. 1984, MNRAS, 209, 111

Joseph, R. D. \& Wright, G. S. 1985, MNRAS, 214, 87

Kankare, E., Mattila, S., Ryder, S., Pérez-Torres, M., Alberdi, A., Romero-Canizales, C., Díaz-Santos, T., Väisänen, P., Efstathiou, A., Alonso-Herrero, A., Colina, L., \& Kotilainen, J. 2008, ApJ, 689, L97

Kennicutt, Jr., R. C. \& Keel, W. C. 1984, ApJ, 279, L5

Krumholz, M. R., Cunningham, A. J., Klein, R. I., \& McKee, C. F. 2010, ApJ, 713, 1120

Larson, R. B. \& Tinsley, B. M. 1978, ApJ, 219, 46

Lotz, J. M., Primack, J., \& Madau, P. 2004, AJ, 128, 163

Mattila, S., Väisänen, P., Farrah, D., Efstathiou, A., Meikle, W. P. S., Dahlen, T., Fransson, C., Lira, P., Lundqvist, P., Östlin, G., Ryder, S., \& Sollerman, J. 2007, ApJ, 659, L9

Michel-Dansac, L., Lambas, D. G., Alonso, M. S., \& Tissera, P. 2008, MNRAS, 386, L82

Mihos, J. C. \& Hernquist, L. 1994, ApJ, 425, L13

-. 1996, ApJ, 464, 641

Nomoto, K. I., Iwamoto, K., \& Suzuki, T. 1995, Phys. Rep., 256, 173

Paumard, T., Genzel, R., Martins, F., Nayakshin, S., Beloborodov, A. M., Levin, Y., Trippe, S., Eisenhauer, F., Ott, T., Gillessen, S., Abuter, R., Cuadra, J., Alexander, T., \& Sternberg, A. 2006, ApJ, 643, 1011

Petrosian, A., Navasardyan, H., Cappellaro, E., McLean, B., Allen, R., Panagia, N., Leitherer, C., MacKenty, J., \& Turatto, M. 2005, AJ, 129, 1369

Petrosian, A. R. \& Turatto, M. 1995, A\&A, 297, 49

Portegies Zwart, S., McMillan, S., Gieles, M. 2010, ArXiv e-prints

Richardson, D., Branch, D., \& Baron, E. 2006, AJ, 131, 2233

Richardson, D., Branch, D., Casebeer, D., Millard, J., Thomas, R. C., \& Baron, E. 2002, AJ, 123, 745

Rieke, G. H., Lebofsky, M. J., Thompson, R. I., Low, F. J., \& Tokunaga, A. T. 1980, ApJ, 238, 24

Rieke, G. H., Loken, K., Rieke, M. J., \& Tamblyn, P. 1993, ApJ, 412, 99

Rupke, D. S. N., Kewley, L. J., \& Barnes, J. E. 2010, ApJ, 710, L156

Salpeter, E. E. 1955, ApJ, 121, 161

Sanders, D. B., Soifer, B. T., Elias, J. H., Madore, B. F., Matthews, K., Neugebauer, G., \& Scoville, N. Z. 1988, ApJ, 325, 74

Shaw, R. L. 1979, A\&A, 76, 188

Smartt, S. J. 2009, ARA\&A, 47, 63 
Stolte, A., Grebel, E. K., Brandner, W., \& Figer, D. F. 2002, A\&A, 394, 459

Tremonti, C. A., Heckman, T. M., Kauffmann, G., Brinchmann, J., Charlot, S., White, S. D. M., Seibert, M., Peng, E. W., Schlegel, D. J., Uomoto, A., Fukugita, M., \& Brinkmann, J. 2004, ApJ, 613, 898

van den Bergh, S. 1997, AJ, 113, 197

Wainwright, C., Berger, E., \& Penprase, B. E. 2007, ApJ, 657, 367

Woosley, S. E. \& Bloom, J. S. 2006, ARA\&A, 44, 507 